\title{
Targeted NBUVB Phototherapy in Childhood Vitiligo: A Study in 35 Children
} Imran Majid ${ }^{1,2^{*}}$

${ }^{1}$ Associate Professor Dermatology, Govt Medical College, Srinagar Kashmir, India

${ }^{2}$ Consultant CUTIS Skin Institute, Srinagar Kashmir, India

*Corresponding author: Dr Imran Majid, CUTIS Skin Institute, Landmark Plaza, Karanagar Chwok, Srinagar Kashmir, India-190010, Tel: 91-9906590822; E-mail: imran54@yahoo.com

Rec date: April 14, 2014; Acc date: May 05, 2014; Pub date: May 07, 2014

Copyright: (c) 2014 Majid I, This is an open-access article distributed under the terms of the Creative Commons Attribution License, which permits unrestricted use, distribution, and reproduction in any medium, provided the original author and source are credited.

\section{Abstract}

Background: Targeted UVB phototherapy currently ranks among the most effective treatment options in localized vitiligo.

Purpose: The study aims to assess the efficacy of Targeted NBUVB monotherapy in childhood vitiligo involving less than $10 \%$ body surface area.

Methods: Thirty-five children with vitiligo, less than 15-years of age were treated with Targeted NBUVB device twice weekly till complete resolution of the treated lesions or till maximum of 30 doses whichever occurred earlier. The extent of re-pigmentation achieved was assessed on the basis of VASI scoring system and was graded as excellent $(>75 \%$ re-pigmentation), good $(50-75 \%$ re-pigmentation) or poor $(<50 \%$ re-pigmentation) at the end of study period. The extent of re-pigmentation achieved was also correlated with the site and duration of vitiligo.

Results: Three patients dropped out of the study after receiving just 1 to 3 doses because of personal reasons. Of the remaining 32 patients there were a total of 22 responders $(68.7 \%)$ who showed at least $50 \%$ repigmentation of treated lesions. A total of 114 lesions were treated out of which 61 lesions $(53.5 \%)$ achieved $>75 \%$ repigmentation. Among the rest, 33 lesions (28.9\%) failed to show a satisfactory response to treatment while 20 lesions $(17.5 \%)$ showed a good response in the form of $50-75 \%$ repigmentation. Lesions on face and neck responded the best to the treatment regimen.

Conclusions: Targeted NBUVB therapy is a promising therapy in childhood vitiligo where the disease involves $<10 \%$ body surface area.

Keywords: Vitiligo; Childhood vitiligo; Treatment; Phototherapy; Targeted UVB phototherapy

\section{Introduction}

Vitiligo is an acquired disorder of melanin pigmentation that affects about $1 \%$ of the world population [1]. The onset of this disease is seen before 15-years of age in majority of cases. Children affected with vitiligo suffer from psychological trauma with a profound effect on their quality of life $[2,3]$.

On the therapeutic front, phototherapy with NBUVB has emerged as one of the most favoured treatment options in vitiligo. This treatment option has been found to be effective in both adult as well as childhood vitiligo with no significant adverse effects [4,5]. Even combination therapies of NBUVB with topical and oral agents have been described with possible synergistic effects [6-8]. Targeted phototherapy is another advancement in this field wherein the phototherapeutic device specifically 'targets' the lesional skin while the rest of skin remains unexposed. Targeted phototherapy offers a number of advantages over whole-body NBUVB treatment as the photo-exposure remains strictly limited to the affected skin allowing higher energies to be delivered and minimizing the adverse effects of cumulative UVB irradiation on the body [8,9]. Additionally, in comparison with traditional NBUVB therapy, Targeted phototherapy needs to be administered less frequently and the therapeutic results are obtained more rapidly as well [10,11]. A number of targeted phototherapeutic devices have been used till date in vitiligo with encouraging results. These include $\mathrm{XeCl}$ excimer laser and lamp and targeted UVB devices delivering broadband UVB (BBUVB) or NBUVB light [11-14]. Targeted UV light-based therapies employ conventional noncoherent ultraviolet light that is delivered through fibre-optic cable systems specifically to the treatment area. These machines deliver the light energy in seconds and have larger spot sizes [14-17].

Targeted phototherapy has been used successfully in childhood vitiligo as well. Clinical studies have demonstrated a good therapeutic effect of excimer laser monotherapy in childhood Vitiligo [18]. Excimer laser has even been used in combination with topical pimecrolimus in childhood vitiligo with good results [19].

\section{Material and Methods}

Thirty-five children, with vitiligo involving less than $10 \%$ body surface area, were enrolled for the present study at our institute from May 2012 to August 2012. All the affected children were less than 15years of age and had a 'stable' disease at the time of enrolment into the study. For the purpose of the present study 'stable vitiligo' was defined as 'vitiligo showing no further spread or spontaneous re-pigmentation 
over a 3-month period'. If the patients were on any topical treatments, they were given a wash-out period of 3-weeks before enrolment into the study. Children who had received any other form of phototherapy in the past and those who had used any immunosuppressant's or immunomodulator drugs for vitiligo over the previous 1-month were excluded from the study. Additionally, children with photosensitive disorders and those with acrofacial vitiligo were also excluded. Acral lesions were excluded as they have been shown to be non-responsive to any form of phototherapy in clinical studies.

All the enrolled children were treated with a targeted phototherapy device named Levia ${ }^{\mathrm{R}}$ Professional Targeted UVB machine (Lerner Medical Devices Inc, USA) that delivers NBUVB light over $300 \mathrm{~nm}$ to $320 \mathrm{~nm}$ with a peak at $314 \mathrm{~nm}$. The device delivers targeted NBUVB light in the range of $5 \mathrm{~mJ} / \mathrm{cm}^{2}$ to $800 \mathrm{~mJ} / \mathrm{cm}^{2}$ with a spot size of 3 square centimeters. The machine comes with a stencil of different shapes and sizes meant to treat similar-shaped or sized lesions. The machine also provides a separate attachment $\left(\right.$ Litebrush ${ }^{\mathrm{R}}$ ) to treat hairy areas like the scalp and chest/back in males.

All the enrolled children were given twice-weekly exposures with a gap of at least 48 hours in between two exposures. Lesions anywhere on the body including those on the eyes or genitals were treated but acral lesions were excluded as described above.

The initial dose used in every patient was $200 \mathrm{~mJ} / \mathrm{cm}^{2}$ and the energy was increased by $50 \mathrm{~mJ} / \mathrm{cm}^{2}$ after every dose till a faint erythema was obtained. The same dose was then continued till the patient continued to show mild erythema or perifollicular pigmentation. In case of eyelids and genital areas, the initial dose used was $100 \mathrm{~mJ} / \mathrm{cm}^{2}$.

In case of painful erythema or blistering at any site, Targeted NBUVB treatment was stopped for 1 week and the dose was reduced by $50 \mathrm{~mJ} / \mathrm{cm}^{2}$ from the last dose received. No topical or oral drug therapy was allowed during the study period. All the children enrolled were followed up weekly for any evidence of re-pigmentation both clinically as well as by repeated digital photographs. Treatment was thus continued for a maximum of 30 sessions or complete repigmentation of treated lesions whichever occurred earlier. If there was no evidence of re-pigmentation by the $12^{\text {th }}$ dose of treatment or any koebnerization at the treatment site in the form of extension of vitiligo or any appearance of fresh lesions around the treatment area, Targeted phototherapy was stopped and patients were offered alternate treatment options.

The extent of re-pigmentation achieved was assessed visually in a manner as given in Table 1 . Re-pigmentation of $>75 \%$ was labeled as 'excellent response' while 50-75\% re-pigmentation constituted a 'good response'. Patients achieving $<50 \%$ re-pigmentation were labeled as poor responders. Chi square test was used for assessing the statistical significance of the results obtained and a $\mathrm{p}$ value of $<0.05$ was considered as 'statistically significant'.

\begin{tabular}{|l|l|}
\hline Extent of re-pigmentation & Clinical Observation \\
\hline $0 \%$ & No change in the depigmented area \\
\hline $10 \%$ & Specks of repigmentation or concavity of margins \\
\hline $25 \%$ & Area of repigmentation less than the residual depigmented area \\
\hline $50 \%$ & Area of repigmentation almost equal to that of residual depigmentation \\
\hline $75 \%$ & Area of repigmentation more than the residual depigmented area \\
\hline $90 \%$ & Some specks of depigmentation left \\
\hline $100 \%$ & Complete repigmentation \\
\hline
\end{tabular}

Table 1: Protocol used to calculate the re-pigmentation achieved and grading

\section{Results}

\begin{tabular}{|l|l|l|l|l|}
\hline Site of vitiligo & $\begin{array}{l}\text { Number of } \\
\text { lesions }\end{array}$ & $\begin{array}{l}\text { Excellent } \\
\text { response }\end{array}$ & $\begin{array}{l}\text { Good } \\
\text { response }\end{array}$ & Poor response \\
\hline $\begin{array}{l}\text { Face and } \\
\text { neck }\end{array}$ & 48 & $35(72.9 \%)$ & $7(14.6 \%)$ & $6(12.5 \%)$ \\
\hline Trunk & 34 & $18(53 \%)$ & $7(20.6 \%)$ & $9(26.4 \%)$ \\
\hline Upper Limbs & 6 & $2(33 \%)$ & 0 & $4(67 \%)$ \\
\hline Lower limbs & 26 & $6(23.1 \%)$ & $6(23.1 \%)$ & $14(53.8 \%)$ \\
\hline Total & 114 & $61(53.5 \%)$ & $20(17.5 \%)$ & $33(29 \%)$ \\
\hline P value--<0.01 &
\end{tabular}

Table 2: Association of response with the site of vitiligo
Three patients dropped out of the study after receiving just 1 to 3 doses. These patients could not continue twice weekly visits to our institute for treatment for personal reasons. Therefore, at the end of study period we had 32 children available for analysis of results (Table 2). The age of enrolled children ranged from 6 years to 15 years with a mean of 11.7 years. Female sex was represented more as we had 20 females in comparison with only 12 males in the study group.

Most of the patients were suffering from Vitiligo vulgaris and there were only 2 patients with segmental type of vitiligo (involving the face). Duration of vitiligo ranged from 6 months to 5 years with a mean of 2.3 years.

No serious adverse events were noted in any patient. Four patients developed intense mildly painful erythema, without blistering, at the treatment site. In all of these patients, treatment was stopped for one week and reintroduced at lower dose without any further adverse events. 
In total, there were 22 responders exhibiting $>50 \%$ re-pigmentation at the treated sites. This accounted for $68.7 \%$ of all the cases available for analysis at the end of study period. Ten patients (31.3\%) did not show a satisfactory response to treatment and were labeled as nonresponders.

Number of lesions treated in each patient ranged from 1 to 8 . A total of 114 treated lesions were thus treated. Of these 48 lesions were located on the face and neck area, 34 on trunk, 6 on upper limbs and
26 on lower limbs. Acral areas, as mentioned earlier, were not treated and were thus excluded from the study analysis.

Out of the 48 lesions present on the face and neck, 35 lesions (72.9\%) achieved $>75 \%$ re-pigmentation (excellent response) while 7 others showed a good response with $50-75 \%$ re-pigmentation (Figure 1a and $1 \mathrm{~b}$ ). Thus only 6 lesions on the head and neck area failed to show a satisfactory response to Targeted NBUVB out of which 2 lesions were those of segmental vitiligo (Table 2).

\begin{tabular}{|l|l|l|l|l|l|}
\hline Duration of vitiligo & Number of patients & Number of lesions & Excellent response & Good response & Poor response \\
\hline 6-12 months & 8 & 18 & $12(67 \%)$ & 0 & $6(33 \%)$ \\
\hline $1-3$ years & 11 & 41 & $22(53.6 \%)$ & $8(19.5 \%)$ & $11(26.8 \%)$ \\
\hline$>3$ years & 13 & 55 & $27(49.1 \%)$ & $12(21.8 \%)$ & $16(29.1 \%)$ \\
\hline Total & 32 & 114 & $61(53.5 \%)$ & $20(17.5 \%)$ & $33(29 \%)$ \\
\hline P value-0.31 & \multicolumn{5}{|l}{} \\
\hline
\end{tabular}

Table 3: Association of response with the duration of vitiligo

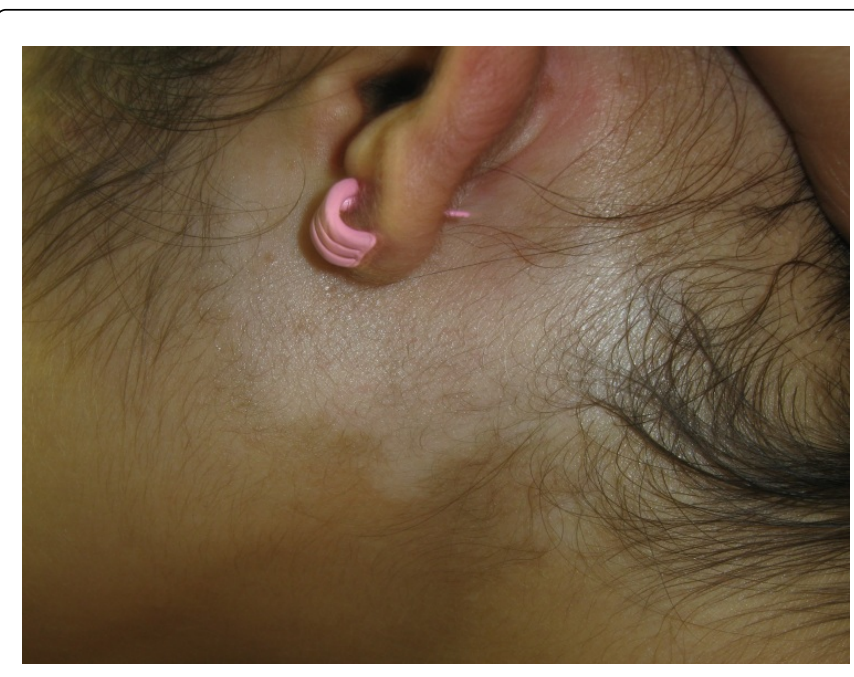

Figure 1a: Vitiligo on post-auricular area in a child

On the trunk, a total of 34 lesions were treated out of which excellent response was seen in 18 lesions (53\%) while 7 and 9 lesions achieved a good and poor response respectively (Table 3 ). Similarly, out of the 26 lesions treated on lower limbs, 6 lesions showed excellent response (23.1\%) while 6 and 14 lesions showed good and poor response respectively. Lesions involving the upper limbs were underrepresented in this study and out of 6 lesions treated, 2 lesions achieved $75-100 \%$ re-pigmentation while the 4 others failed to show any significant response. Thus, out of 114 lesions treated on different body areas, excellent response was observed in 61 lesions (53.5\%) while 20 others $(17.5 \%)$ showed a good response. In total 52 lesions (45.6\%) exhibited total or near-total re-pigmentation (Figure $2 \mathrm{a}$ and $2 b)$. No significant re-pigmentation $(<50 \%)$ was seen in 33 lesions, accounting for $29 \%$ of the total lesions treated.

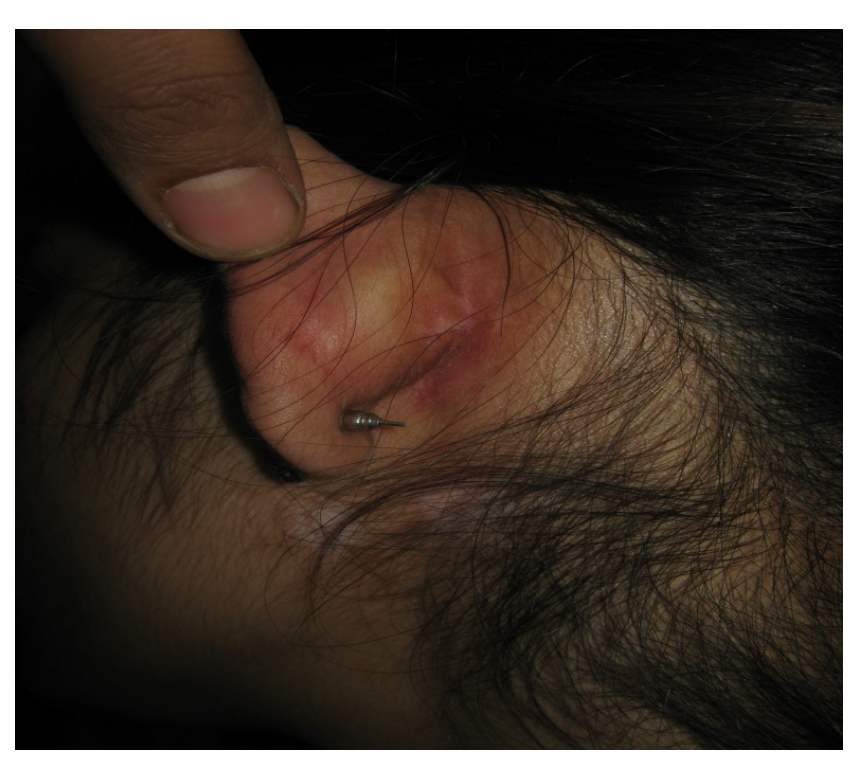

Figure 1b: Excellent response after 14 doses of Targeted NBUVB treatment

No untreated lesion in the study population showed any significant re-pigmentation over the study period and no extension of lesions was seen as well.

Onset of re-pigmentation in treated lesions was seen as early as the 4th dose of treatment in 14 cases. And by the 12th dose all those lesions which eventually re-pigmented, showed some evidence of pigmentation, either marginal or as perifollicular islands.

Number of Targeted NBUVB doses required to achieve complete re-pigmentation of the treated lesions ranged from a minimum of 8 doses given over 4 weeks to a maximum of 26 doses administered over a period of about 14 weeks. Thus, in some of the study cases the treatment was discontinued after just over a month because the treated 
lesion had repigmented completely within this time. No child who ultimately achieved a complete re-pigmentation of the treated lesion needed more than 26 doses to do so.

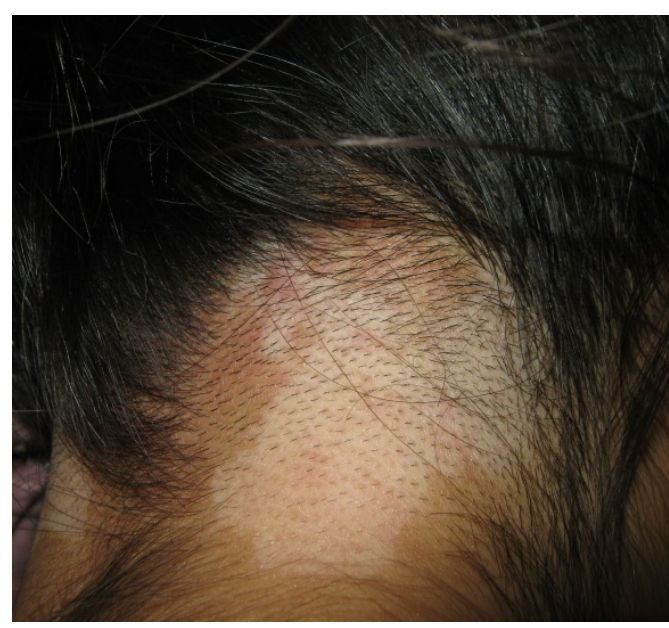

Figure 2a: Vitiligo on neck in a 14-year old female

An attempt was made to correlate the response achieved with the site of the treated lesions and the duration of vitiligo. Lesions on the face and neck area were seen to respond the best to the treatment regimen followed by trunk and lower limbs. While $72.9 \%$ of lesions achieved excellent response on face and neck area, the corresponding figure was $53 \%$ and $25 \%$ on the trunk and limbs respectively (Table 3 ).
The difference in the response between the different sites was tested for its statistical significance by means of chi-square test and the difference was found to be statistically significant (chi square value of 21.48 and $p$ value of $<0.001$ ).

The response to treatment was also correlated with the duration of vitiligo. Re-pigmentation achieved in patients with different durations of vitiligo is shown in Table 4. Difference in the response between the three groups was tested statistically by using the chi square test and was not found to be statistically significant ( with a chi square value of 4.78 and $p$ value of 0.310 ).

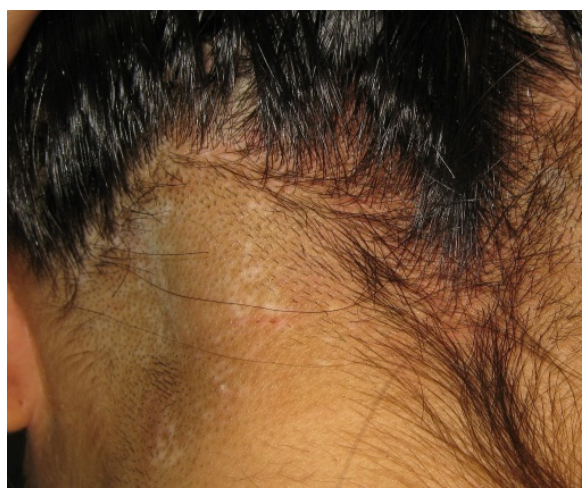

Figure 2b: Excellent re-pigmentation after 24 doses of Targeted phototherapy

\begin{tabular}{|c|c|c|c|c|}
\hline Study & Population & Device used & Overall response & Additional remarks \\
\hline Cho et al. [18] & Children & Excimer laser & $\begin{array}{l}50 \% \text { repigmentation in } 17 \text { out of } 30 \text { patients } \\
(56.7 \%)\end{array}$ & $\begin{array}{l}\text { Best response on face, neck } \\
\text { and trunk }\end{array}$ \\
\hline Lan et al. [19] & Children & $\begin{array}{l}\text { Excimer laser with or } \\
\text { without pimecrolimus }\end{array}$ & $\begin{array}{l}71 \% \text { patients achieved }>50 \% \text { repigmentation with } \\
\text { combination; } 50 \% \text { patients with excimer laser } \\
\text { alone }\end{array}$ & $\begin{array}{lll}\text { Better response } & \text { with } \\
\text { combination } & \text { than } & \text { with } \\
\text { monotherapy } & & \end{array}$ \\
\hline Menchini et al. [21] & Adults & Bioskin & $\begin{array}{l}>75 \% \text { repigmentation in } 69.48 \% \text { of the } 734 \\
\text { patients treated over } 12 \text { months, complete } \\
\text { repigmentation in } 15.25 \% \text { patients }\end{array}$ & 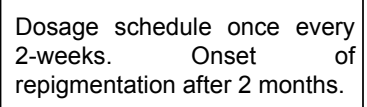 \\
\hline Asawanonda et al. [16] & Adults & B-clear & $\begin{array}{l}29 \text { vitiligo lesions in } 6 \text { patients achieved good } \\
\text { response }\end{array}$ & $\begin{array}{l}\text { Onset of repigmentation within } \\
\text { 3-weeks. Site dependent. }\end{array}$ \\
\hline Welsh et al. [14] & Adults & Targeted Broadband UVB & $\begin{array}{l}\text { Average repigmentation of } 66.25 \% \text { on face and } \\
31.25 \% \text { on trunk, neck and genitalia. }\end{array}$ & $\begin{array}{l}\text { Onset of repigmentation after } \\
16-20 \text { sessions. }\end{array}$ \\
\hline
\end{tabular}

Table 4: Comparison with other studies on Targeted UVB in children and adults

Children who responded to treatment were followed up for a period of 3 months after the end of treatment regimen to look for the stability of pigmentation achieved. None of them showed any loss of pigmentation over the treated areas in the follow up period.

\section{Discussion}

Childhood vitiligo is always a challenge to manage as many of the treatment options that can be safely and easily used in adult population are either unsafe or impossible to use in children. Phototherapy with whole-body NBUVB chambers is practically difficult and at times impossible to administer in children especially at a young age. This disadvantage is not shared by Targeted phototherapy which is administered in a much more comfortable manner than traditional NBUVB therapy. Moreover, Targeted phototherapy also offers certain therapeutic advantages over wholebody NBUVB treatment especially for localized vitiligo [10,12,13]. Among targeted phototherapy devices currently available, excimer laser has been shown to induce the most rapid onset of repigmentation in vitiligo [11]. This phototherapeutic device has been shown to be effective in localized childhood vitiligo as well $[18,19]$. In addition to excimer laser a monochromatic excimer lamp has also 
been utilized in the treatment of vitiligo with almost comparable results [12].

As far as the targeted UVB devices are concerned, the UV spectrum delivered varies from one machine to another. These devices include 'BClear' that delivers broadband UVB, 'Multiclear' or 'Dualight' providing UVA and UVB combination and lastly 'Bioskin' which gives a NBUVB waveband peaking at $311 \mathrm{~nm}$ [14-16]. Clinical studies have demonstrated that the results obtained are similar with the BB-UVB and NBUVB Targeted phototherapy devices in vitiligo [17]. In a clinical study conducted by Aswanonda et al., the overall response to the two different wavebands was seen to be similar at 12 weeks and this similarity was then maintained even at 24th week of treatment [17]. Targeted phototherapy with NBUVB is also known as 'microphototherapy'. The efficacy of this form of phototherapy has been demonstrated in both segmental as well as non-segmental vitiligo in adults [20,21]. However, different authors have used different treatment schedules ranging from twice-weekly to once every two weeks treatment schedules. Both these treatment schedules have eventually led to significant therapeutic benefit in treated patients $[20,21]$.

Studies on targeted phototherapy in childhood vitiligo are limited in number. In a study on excimer laser in childhood vitiligo, repigmentation of $>50 \%$ was seen in 17 out of 30 patients $(56.7 \%)$ treated with this modality [18]. Moreover, the response was seen to be site dependent, favoring the face, neck and trunk. No significant adverse effects were observed in this study.

Another study excimer laser monotherapy was compared with a combination of topical pimecrolimus and excimer laser treatment. In this study $71 \%$ patients achieved Grade 3 or 4 re-pigmentation with the combination treatment while only $50 \%$ patients achieved the same level of therapeutic benefit with excimer laser monotherapy [19].

We have treated 35 children with NBUVB based targeted phototherapy in this study and we could achieve an excellent response in the form of $>75 \%$ re-pigmentation in $53.5 \%$ of treated lesions. In addition, $17.5 \%$ more lesions showed a partial re-pigmentation of $50-75 \%$ taking the total percentage of responding lesions to $71 \%$. This percentage is better than those quoted by earlier studies on Targeted phototherapy in childhood vitiligo $[18,19,22]$. We followed a twiceweekly schedule in our patients which is the one followed by majority of studies on Targeted phototherapy.

We could achieve an earlier onset of re-pigmentation in our patients, as early as at $4^{\text {th }}$ dose. This is more in conformity with the results achieved in vitiligo with excimer laser phototherapy than with the Targeted UVB devices described above. This difference can be explained probably on the basis of the difference in treatment schedule followed.

As with earlier studies, response to Targeted phototherapy in our study was seen to be site dependent with the head and neck regions responding the best to this modality (Table 4). This observation has been shared by almost all the clinical studies on this subject.

We did not treat acral lesions and we had just two patients of segmental vitiligo in our group. So the comparison between the therapeutic responses in different morphological types of vitiligo cannot be commented upon.

An important observation from our study was that any patient not showing any re-pigmentation to targeted UVB phototherapy by the $10^{\text {th }}$ or $12^{\text {th }}$ dose eventually showed a poor response to treatment. This observation is highly relevant clinically as it signifies that a patient is unlikely to respond to Targeted phototherapy if there is no evidence of re-pigmentation by the end of 6 weeks of treatment. Therefore, while administering this form of treatment in vitiligo, a period of 6-weeks should be enough to gauge whether a patient is expected to respond to treatment or not. Continuing the treatment beyond six weeks in such cases is highly unlikely to yield any positive results.

\section{Conclusions}

Targeted NBUVB phototherapy is an effective treatment option in childhood vitiligo with a rapid onset of re-pigmentation. The response to treatment is dependent on the site of the vitiligo lesion. Onset of repigmentation is expected to manifest within a maximum of 6 weeks.

\section{References}

1. Krüger C1, Schallreuter KU (2012) A review of the worldwide prevalence of vitiligo in children/adolescents and adults. Int J Dermatol 51: 1206-1212.

2. Ongenae K, Beelaert L, van Geel N, Naeyaert JM (2006) Psychosocial effects of vitiligo. J Eur Acad Dermatol Venereol 20: 1-8.

3. Kent G, al-Abadie M (1996) Factors affecting responses on Dermatology Life Quality Index items among vitiligo sufferers. Clin Exp Dermatol 21: 330-333.

4. Scherschun L, Kim JJ, Lim HW (2001) Narrow-band ultraviolet B is a useful and well-tolerated treatment for vitiligo. J Am Acad Dermatol 44: 999-1003.

5. Njoo MD, Bos JD, Westerhof W (2000) Treatment of generalized vitiligo in children with narrow-band (TL-01) UVB radiation therapy. J Am Acad Dermatol 42: 245-253.

6. Majid I (2010) Does topical tacrolimus ointment enhance the efficacy of narrowband ultraviolet $\mathrm{B}$ therapy in vitiligo? A left-right comparison study. Photodermatol Photoimmunol Photomed 26: 230-234.

7. Esfandiarpour I, Ekhlasi A, Farajzadeh S, Shamsadini S (2009) The efficacy of pimecrolimus $1 \%$ cream plus narrow-band ultraviolet $B$ in the treatment of vitiligo: a double-blind, placebo-controlled clinical trial. J Dermatolog Treat 20: 14-18.

8. Schallreuter KU, Wood JM, Lemke KR, Levenig C (1995) Treatment of vitiligo with a topical application of pseudocatalase and calcium in combination with short-term UVB exposure: a case study on 33 patients. Dermatology 190: 223-229.

9. Grimes PE (2003) Advances in the treatment of vitiligo: Targeted phototherapy. Cosm Dermatol 16:18-22.

10. Hamzavi IH, Lim HW, Syed ZU (2012) Ultraviolet-based therapy for vitiligo: what's new? Indian J Dermatol Venereol Leprol 78: 42-48.

11. Park KK, Liao W, Murase JE (2012) A review of monochromatic excimer light in vitiligo. Br J Dermatol 167: 468-478.

12. Le Duff F, Fontas E, Giacchero D, Sillard L, Lacour JP, et al. (2010) 308$\mathrm{nm}$ excimer lamp vs. 308-nm excimer laser for treating vitiligo: a randomized study. Br J Dermatol 163: 188-192.

13. Choi KH, Park JH, Ro YS (2004) Treatment of Vitiligo with 308-nm xenon-chloride excimer laser: Therapeutic efficacy of different initial doses according to treatment areas. J Dermatol 31:284-92.

14. Welsh O, Herz-Ruelas ME, Gómez M, Ocampo-Candiani J (2009) Therapeutic evaluation of UVB-targeted phototherapy in vitiligo that affects less than $10 \%$ of the body surface area. Int J Dermatol 48: 529-534.

15. Akar A, Tunca M, Koc E, Kurumlu Z (2009) Broadband targeted UVB phototherapy for localized vitiligo: a retrospective study. Photodermatol Photoimmunol Photomed 25: 161-163.

16. Asawanonda P, Charoenlap M, Korkij W (2006) Treatment of localized vitiligo with targeted broadband UVB phototherapy: a pilot study. Photodermatol Photoimmunol Photomed 22: 133-136. 
Citation: Majid I (2014) Targeted NBUVB Phototherapy in Childhood Vitiligo: A Study in 35 Children . Pigmentary Disorders 1: 101. doi:10.4172/ jpd.1000101

Page 6 of 6

17. Asawanonda P, Kijluakiat J, Korkij W, Sindhupak W (2008) Targeted Broadband Ultraviolet B Phototherapy produces similar responses to Targeted Narrowband Ultraviolet B phottherapy for vitiligo: A randomized, double-blind study. Acta Dermatol Venereol 88:376-381.

18. Cho S, Zheng Z, Park YK, Roh MR (2011) The 308-nm excimer laser: a promising device for the treatment of childhood vitiligo. Photodermato Photoimmunol Photomed 27: 24-29.

19. Hui-Lan Y, Xiao-Yan H, Jian-Yong F, Zong-Rong L (2009) Combination of 308-nm excimer laser with topical pimecrolimus for the treatment of childhood vitiligo. Pediatr Dermatol 26: 354-356.
20. Lotti TM, Menchini G, Andreassi L (1999) UV-B radiation microphototherapy. An elective treatment for segmental vitiligo. J Eur Acad Dermatol Venereol 13: 102-108.

21. Menchini G, Tsoureli-Nikita E, Hercogova J (2003) Narrow-band UV-B micro-phototherapy: a new treatment for vitiligo. J Eur Acad Dermatol Venereol 17: 171-177.

22. Percivalle S, Piccinno R, Caccialanza M, Forti S (2012) Narrowband ultraviolet $\mathrm{B}$ phototherapy in childhood vitiligo: evaluation of results in 28 patients. Pediatr Dermatol 29: 160-165. 